Journal of Applied Mathematics and Computing manuscript No.

(will be inserted by the editor)

\title{
Global dynamics of a delayed SIRS epidemic model with a wide class of nonlinear incidence rates
}

\author{
Yoichi Enatsu - Eleonora Messina - Yukihiko \\ Nakata · Yoshiaki Muroya - Elvira Russo · \\ Antonia Vecchio
}

Received: date / Accepted: date

\begin{abstract}
In this paper, by constructing Lyapunov functionals, we consider the global dynamics of an SIRS epidemic model with a wide class of nonlinear incidence rates and distributed delays $\int_{0}^{h} p(\tau) f(S(t), I(t-\tau)) d \tau$ under the condition that the total population $N(t)$ converges to 1 . By using a technical lemma which is derived from strong condition of strict monotonicity of functions $f(S, I)$ and $f(S, I) / I$ with respect to $S \geq 0$ and $I>0$, we extend the global stability result for an SIR epidemic model if $R_{0}>1$, where $R_{0}$ is the basic reproduction number. By using a limit system of the model, we also give a proof that the disease-free equilibrium is globally asymptotically stable for $R_{0}=1$.
\end{abstract}

Keywords SIRS epidemic model $\cdot$ nonlinear incidence rate $\cdot$ global asymptotic stability · Lyapunov functional

Mathematics Subject Classification (2000) 34K20 -34K25 92 D30

Yoichi Enatsu

Department of Pure and Applied Mathematics, Waseda University, Ohkubo 3-4-1, Shinjuku-ku, Tokyo 1698555, Japan

E-mail: yo1.gc-rw.docomo@akane.waseda.jp

Eleonora Messina, Elvira Russo

Dipartimento di Matematica e Applicazioni, Università degli Studi di Napoli "Federico II"-Via Cintia, I80126 Napoli, Italy

E-mail: eleonora.messina@unina.it, elvrusso@unina.it

Yukihiko Nakata

Basque Center for Applied Mathematics, Bizkaia Technology Park, Building 500 E-48160 Derio, Spain

E-mail: nakata@bcamath.org

Yoshiaki Muroya

Department of Mathematics, Waseda University, Ohkubo 3-4-1, Shinjuku-ku, Tokyo 169-8555, Japan

E-mail: ymuroya@waseda.jp

Antonia Vecchio

Ist. per Appl. del Calcolo "M. Picone", Sede di Napoli-CNR-Via P. Castellino, 111-80131 Napoli- Italy

E-mail: a.vecchio@iac.cnr.it 


\section{Introduction}

In order to understand the mechanism of disease transmission, many authors have paid attention to the stability analysis of the equilibria for various kinds of epidemic models (see [1-24] and the references therein).

To investigate the disease spreading effect transmitted by a vector (e.g. mosquitoes, rats, etc.) after an incubation time denoting the time during which the infectious agents develop in the vector, Takeuchi et al. [21] formulated an SIR (Susceptible-Infected-Recovered) epidemic model with distributed delays of the form $\beta S(t) \int_{0}^{h} p(\tau) I(t-\tau) d \tau$. However, when the reproduction number is larger than 1 , it was shown that the endemic equilibrium is globally asymptotically stable only for the case that the delay $h$ is small enough, that is, an open problem for the global stability of the endemic equilibrium for the case $h$ is sufficiently large was still left. Later, by focusing on the equation deformation in time derivative of a Lyapunov functional, McCluskey [14] proved that the endemic equilibrium is globally asymptotically stable for any length of delay $h$ if the basic reproduction number is larger than 1. Recently, Enatsu et al. [5] and McCluskey [16] considered the following SIR epidemic model with a wide class of nonlinear incidence rates and distributed delays:

$$
\left\{\begin{array}{l}
\frac{\mathrm{d} S(t)}{\mathrm{d} t}=\mu-\mu S(t)-\int_{0}^{h} p(\tau) f(S(t), I(t-\tau)) d \tau \\
\frac{\mathrm{d} I(t)}{\mathrm{d} t}=\int_{0}^{h} p(\tau) f(S(t), I(t-\tau)) d \tau-(\mu+\gamma) I(t) \\
\frac{\mathrm{d} R(t)}{\mathrm{d} t}=\gamma I(t)-\mu R(t)
\end{array}\right.
$$

$S(t), I(t)$ and $R(t)$ denote the fractions of susceptible, infective and recovered individuals at time $t$, respectively. $\mu>0$ is the death rate of the population, $\gamma>0$ is the recovery rate of the infective individuals. $h>0$ is a maximum time taken to become infectious and the transmission of the infection is governed by an incidence rate $\int_{0}^{h} p(\tau) f(S(t), I(t-\tau)) d \tau$. $p(\tau)$ denotes the fraction of vector population in which the time taken to become infectious is $\tau \in[0, h]$ satisfying $p \in C\left([0, h], \mathbb{R}_{+0}\right)$ and $\int_{0}^{h} p(\tau) d \tau=1$.

By constructing suitable Lyapunov functionals, they showed that the global stability of equilibria of (1.1) is fully determined by the basic reproduction number when the functions $f(S, I)$ and $f(S, I) / I$ has monotone properties with respect to $S \geq 0$ and $I>0$. The similar results have now been obtained in Huang and Takeuchi [8] for an SIR epidemic model with the wide class of nonlinear incidence rates and a discrete delay.

On the other hand, Mena-Lorcat and Hethcote [17] considered several SIRS (SusceptibleInfected-Recovered-Susceptible) epidemic models with a bilinear incidence rate and a standard incidence rate. The SIRS models describe the phenomena that susceptible individuals become infectious, then removed with immunity after recovery from infection and then susceptible again when the temporary immunity fades away. In order to investigate the effect of the immunity loss of diseases, various kinds of SIRS epidemic models and a significant body of work concerning the stability analysis of the steady states of the models have now been carried out (see, for example, [1,9-12,22] and the references therein).

In addition, many authors have suggested that transmission of the infection shall have a nonlinear incidence rate. Capasso and Serio [3] studied the cholera epidemic spread in Bari in 1973 and have given an assumption that the incidence rate may take the nonlinear form $\frac{\beta S(t) I(t)}{1+\alpha I(t)}$, which has been interpreted as saturated incidence rate measuring the crowding effect of the infective individuals (see also $\mathrm{Xu}$ and $\mathrm{Ma}$ [23]). Based on their idea, Ko- 
robeinikov and Maini [10] and Korobeinikov [11,12] obtained the global properties of SIR and SEIR models with a nonlinear incidence rate $h(S(t)) g(I(t))$ and SIR, SEIR and SIRS epidemic models with a more general framework of the incidence rate $f(S(t), I(t))$, respectively. Thereafter, Zhou and Cui [24] have introduced a nonlinear incidence rate of the form $\beta S(t) I(t)\left(1+\alpha I(t)^{k-1}\right)$ with $k=2$ for an SEIV epidemic model.

However, for global stability conditions of the endemic equilibrium of the delayed SIRS epidemic model, only restricted sufficient conditions are known by literatures. For the model with a bilinear incidence rate, Nakata et al. [20] obtained a sufficient condition which ensure the global stability of the endemic equilibrium. Vargas-De-León and Gómez-Alcaraz [22] constructed an another Lyapunov functional for the same global stability result. For the model with nonlinear incidence rates, Enatsu et al. [6] also obtained that the endemic equilibrium for an SIRS epidemic model with an incidence rate of the form $\int_{0}^{h} \beta S(t) G(I(t-\tau)) d \tau$ is globally stable for a small rate of immunity loss by constructing a Lyapunov functional. By improving monotone iterative techniques in $\mathrm{Xu}$ and Ma [23], Muroya et al. [19] obtained sufficient conditions which ensure the global asymptotic stability of an endemic equilibrium for an SIRS epidemic model with an incidence rate $\beta S(t) I(t-\tau) /\left(1+\alpha I(t-\tau)^{p}\right)$, where $p \geq 1$ (see also Muroya et al. [18] for $p=2$ ). In fact, by introducing a generalized nonlinear incidence as a function of the number of infected individuals, Alexander and Moghadas [1] showed that stability of the endemic equilibrium can change through Hopf, saddle-node and Bogdanov-Takens bifurcations.

Motivated by the above facts, in this paper, we extend global stability results in $[5,6,8$, 16] to the following SIRS epidemic model with a wide class of the nonlinear incidence rates and distributed delays:

$$
\left\{\begin{array}{l}
\frac{\mathrm{d} S(t)}{\mathrm{d} t}=\mu-\mu S(t)-\int_{0}^{h} p(\tau) f(S(t), I(t-\tau)) d \tau+\delta R(t), \\
\frac{\mathrm{d} I(t)}{\mathrm{d} t}=\int_{0}^{h} p(\tau) f(S(t), I(t-\tau)) d \tau-(\mu+\gamma) I(t) \\
\frac{\mathrm{d} R(t)}{\mathrm{d} t}=\gamma I(t)-(\mu+\delta) R(t)
\end{array}\right.
$$

with the initial condition

$$
\left\{\begin{array}{l}
S(\theta)=\varphi_{1}(\theta), I(\theta)=\varphi_{2}(\theta), R(\theta)=\varphi_{3}(\theta) \\
\varphi_{i}(\theta) \geq 0, \theta \in[-h, 0], \varphi_{i}(0)>0, \varphi_{i} \in C\left([-h, 0], \mathbb{R}^{+}\right), i=1,2,3
\end{array}\right.
$$

$\delta \geq 0$ is the rate at which recovered individuals lose immunity and return to the susceptible class. We here assume that $f: \mathbb{R}_{+0}^{2} \rightarrow \mathbb{R}_{+0}$ is a locally Lipschitz continuous function on $\mathbb{R}_{+0}^{2}$ satisfying $f(0, I)=f(S, 0)=0$ for $S, I \geq 0$ and the followings hold.

$(\mathrm{H} 1)\left\{\begin{array}{l}\text { i) } f(S, I) \text { is a strictly monotone increasing function of } S \geq 0, \\ \text { for any fixed } I>0, \\ \text { ii) if } R_{0}>1 \text {, then } f(S, I) \text { is a monotone increasing function of } I \geq 0, \\ \text { for any fixed } S \geq 0,\end{array}\right.$

and

(H2) $\left\{\begin{array}{l}\text { i) } \phi(S, I)=\frac{f(S, I)}{I} \text { is a bounded and monotone decreasing function of } \\ I>0, \text { for any fixed } S \geq 0, \\ \text { ii) } K(S) \equiv \lim _{I \rightarrow+0} \phi(S, I) \text { is a continuous and monotone increasing } \\ \text { function on } S \geq 0,\end{array}\right.$ 
where

$$
R_{0}=\frac{K\left(S_{0}\right)}{\mu+\gamma}, \quad S_{0}=1
$$

is the basic reproduction number of system (1.2). $R_{0}$ denotes the expected number of secondary infectious cases generated by one typical primary case in an entirely susceptible and sufficiently large population. We note that $0<\phi(S, I) \leq K(S)$ holds for any $S, I>0$.

It is well known by the fundamental theory of functional differential equations that the solution $(S(t), I(t), R(t))$ of system (1.2) is unique and positive for all $t \geq 0$.

System (1.2) always has a disease-free equilibrium $E_{0}=\left(S_{0}, 0,0\right)$. On the other hand, under the hypotheses (H1) and (H2), if $R_{0}>1$, then system (1.2) also admits a unique positive equilibrium $E_{*}=\left(S^{*}, I^{*}, R^{*}\right)$, where $S^{*}, I^{*}, R^{*}>0$ (see Korobeinikov [11,12] and Lemma 2.2 below).

The main results are as follows:

Theorem 1.1 If $R_{0} \leq 1$, then the disease-free equilibrium $E_{0}$ of system (1.2) is globally asymptotically stable.

By applying a key property of strict monotonicity of functions $f(S, I)$ and $f(S, I) / I$ with respect to $S \geq 0$ and $I>0$, we obtain sufficient conditions which ensure the global asymptotic stability of the endemic equilibrium $E_{*}$ of system (1.2) for $R_{0}>1$.

Theorem 1.2 If $R_{0}>1$, then the endemic equilibrium $E_{*}$ of system (1.2) exists uniquely and system (1.2) is permanent. Moreover, if the following conditions hold:

$$
\left\{\begin{array}{l}
\text { (I) there exist positive constants } C_{0}, C_{1} \text { and } C_{2} \text { such that } \\
\quad \text { for any } v_{1} \leq S \leq S_{0}, 0 \leq I \leq S_{0}, S \leq S^{*} \text { and } I \neq I^{*}, \\
\frac{f\left(S, I^{*}\right)-f\left(S^{*}, I^{*}\right)}{\left(S-S^{*}\right) I^{*}} \leq C_{0}, \\
\frac{f(S, I)-f\left(S, I^{*}\right)}{I-I^{*}} \geq C_{1}>0, \\
\frac{\frac{I}{f(S, I)}-\frac{I^{*}}{f\left(S, I^{*}\right)}}{I-I^{*}} \geq C_{2}>0, \\
\text { (II) } \delta^{2}<4 C_{0} C_{1} C_{2}(\mu+\delta)(\mu+\gamma) \frac{f\left(v_{1}, I^{*}\right)}{I^{*}},
\end{array}\right.
$$

then the endemic equilibrium $E_{*}$ of system (1.2) is globally asymptotically stable, where $v=v_{1}>0$ is a unique positive solution of $\mu-K(v)-\mu v=0$.

Note that if $f(S, I)=\beta S I /(1+\alpha I)$, then the incidence rate becomes saturated-type, which is of the form used in $\mathrm{Xu}$ and $\mathrm{Ma}$ [23].

Theorem 1.2 indicates that the endemic equilibrium of system (1.2) is globally asymptotically stable when $R_{0}>1$ for a small rate of immunity loss $\delta$ as long as the infection rate has suitable monotone properties of function $f$ characterized by $(\mathrm{H} 1),(\mathrm{H} 2)$ and the condition (1.5).

The organization of this paper is as follows. In Section 2, we offer basic results for system (1.2). In Section 3, we establish global asymptotic stability of the disease-free equilibrium for $R_{0} \leq 1$ and prove Theorem 1.1. In Section 4, we establish global asymptotic stability of the endemic equilibrium for $R_{0}>1$ and prove Theorem 1.2 by means of Lyapunov functionals to the reduced system which is derived from system (1.2). Finally, a discussion is offered in Section 6. 


\section{Basic results}

We offer some basic results of system (1.2). The following lemmas will be used in the proofs of Theorems 1.1 and 1.2.

Lemma 2.1 The plane $S(t)+I(t)+R(t)=S_{0}$ is an invariant manifold of system (1.2), which is globally attractive in the first octant of $\mathbb{R}^{3}$, that is,

$$
\lim _{t \rightarrow+\infty}(S(t)+I(t)+R(t))=S_{0}
$$

Proof Let $N(t)=S(t)+I(t)+R(t)$. Then it follows from system (1.2) that

$$
\begin{aligned}
\frac{\mathrm{d} N(t)}{\mathrm{d} t} & =\mu-\mu S(t)-\mu I(t)-\mu R(t) \\
& =\mu-\mu N(t) .
\end{aligned}
$$

Hence, we obtain that $\lim _{t \rightarrow+\infty} N(t)=S_{0}$. This completes the proof.

Lemma 2.2 System (1.2) always has a disease-free equilibrium $E_{0}=\left(S_{0}, 0,0\right)$. Moreover, if $R_{0}>1$, then system (1.2) has a unique endemic equilibrium $E_{*}=\left(S^{*}, I^{*}, R^{*}\right)$ satisfying the following equations:

$$
\left\{\begin{array}{l}
\mu-\mu S^{*}-f\left(S^{*}, I^{*}\right)+\delta R^{*}=0 \\
f\left(S^{*}, I^{*}\right)-(\mu+\gamma) I^{*}=0 \\
\gamma I^{*}-(\mu+\delta) R^{*}=0
\end{array}\right.
$$

Proof First, it is evident that there always exists a disease-free equilibrium $E_{0}$. Second, we now show that system (1.2) has a unique endemic equilibrium $E_{*}=\left(S^{*}, I^{*}, R^{*}\right)$ if $R_{0}>1$. By (2.2), at a fixed point of the system, the following equalities hold.

$$
\mu-\mu S-\left\{(\mu+\gamma)-\frac{\gamma \delta}{\mu+\delta}\right\} I=0, f(S, I)-(\mu+\gamma) I=0 .
$$

By the implicit function theorem and the hypotheses (H1), we see that $f(S, I)-(\mu+\gamma) I=0$ defines a function $S=\zeta(I)$ on neighborhood around $I=0$. Then, it follows from (2.3) that

$$
\lim _{I \rightarrow+0} \frac{f(\zeta(I), I)}{I}=\mu+\gamma<K\left(S_{0}\right)=\lim _{I \rightarrow+0} \frac{f\left(S_{0}, I\right)}{I},
$$

if $R_{0}=K\left(S_{0}\right) /(\mu+\gamma)>1$. Therefore, by the hypotheses $(\mathrm{H} 2)$, we obtain that

$$
\lim _{I \rightarrow+0} \zeta(I)<S_{0}
$$

By the hypothesis $(\mathrm{H} 2)$, the function $\zeta(I)$ is a monotone increasing function and either exists and is continuous for $I \in\left(0, \mu /\left\{(\mu+\gamma)-\frac{\gamma \delta}{\mu+\delta}\right\}\right]$, or reaches infinity in this interval. After substituting the relations $S=\zeta(I)$ and $f(S, I)-(\mu+\gamma) I=0$ into the first equation of (2.3), we consider the following equation.

$$
H(I):=\mu-\mu \zeta(I)-\left\{(\mu+\gamma)-\frac{\gamma \delta}{\mu+\delta}\right\} I=0,
$$


from which we obtain that $H(I)$ is a strictly monotone decreasing function. By (2.5), we have that

$$
\lim _{I \rightarrow+0} H(I)=\mu-\mu \lim _{I \rightarrow+0} \zeta(I)>\mu-\mu S_{0}=0
$$

which implies that there exists a unique positive solution $0<I^{*}<\mu /\left\{(\mu+\gamma)-\frac{\gamma \delta}{\mu+\delta}\right\}<$ $S_{0}=1$ such that $H\left(I^{*}\right)=0$. Therefore, there exists a unique positive solution $R^{*}>0$ such that $\gamma I^{*}-(\mu+\delta) R^{*}=0$. Hence, the proof is complete.

\section{Global stability of the disease-free equilibrium $E_{0}$}

In this section, we assume that $R_{0} \leq 1$ and show the global asymptotic stability of the disease-free equilibrium $E_{0}$ of system (1.2) constructing a Lyapunov functional. From Lemma 2.1 , the limit set of system (1.2) in the first octant of $\mathbb{R}^{3}$ locates on the plane $S+I+R=S_{0}$. Hence, the dynamics of system (1.2) in the first octant of $\mathbb{R}^{3}$ is equivalent to the following system:

$$
\left\{\begin{array}{l}
\frac{\mathrm{d} S(t)}{\mathrm{d} t}=\mu-\mu S(t)-\int_{0}^{h} p(\tau) f(S(t), I(t-\tau)) d \tau+\delta R(t), \\
\frac{\mathrm{d} I(t)}{\mathrm{d} t}=\int_{0}^{h} p(\tau) f(S(t), I(t-\tau)) d \tau-(\mu+\gamma) I(t) . \\
\frac{\mathrm{d} R(t)}{\mathrm{d} t}=\gamma\left(S_{0}-S(t)-R(t)\right)-(\mu+\delta) R(t) .
\end{array}\right.
$$

Theorem 3.1 If $R_{0} \leq 1$, then the disease-free equilibrium $E_{0}$ of system (3.1) is globally asymptotically stable on $\left\{(S, I, R) \in \mathbb{R}_{+}^{3} \mid S+I+R=S_{0}\right\}$.

Proof We consider the following Lyapunov functional:

$$
W(t)=\int_{S_{0}}^{S(t)}\left(1-\frac{K\left(S_{0}\right)}{K(s)}\right) d s+I(t)+\int_{0}^{h} p(\tau) \int_{t-\tau}^{t} \frac{K\left(S_{0}\right)}{K(S(u+\tau))} f(S(u+\tau), I(u)) d u d \tau
$$


We then obtain

$$
\begin{aligned}
\frac{\mathrm{d} W(t)}{\mathrm{d} t}= & \left(1-\frac{K\left(S_{0}\right)}{K(S(t))}\right)\left(\mu-\mu S(t)-\int_{0}^{h} p(\tau) f(S(t), I(t-\tau)) d \tau+\delta R(t)\right) \\
& +\int_{0}^{h} p(\tau) f(S(t), I(t-\tau)) d \tau-(\mu+\gamma) I(t) \\
& +\int_{0}^{h} p(\tau)\left\{\frac{K\left(S_{0}\right)}{K(S(t+\tau))} f(S(t+\tau), I(t))-\frac{K\left(S_{0}\right)}{K(S(t))} f(S(t), I(t-\tau))\right\} d \tau \\
= & \left(1-\frac{K\left(S_{0}\right)}{K(S(t))}\right)\left(-\mu\left(S(t)-S_{0}\right)+\delta R(t)\right) \\
& -\int_{0}^{h} p(\tau)\left(1-\frac{K\left(S_{0}\right)}{K(S(t))}\right) f(S(t), I(t-\tau)) d \tau \\
& +\int_{0}^{h} p(\tau) f(S(t), I(t-\tau)) d \tau-(\mu+\gamma) I(t) \\
& +\int_{0}^{h} p(\tau)\left\{\frac{K\left(S_{0}\right)}{K(S(t+\tau))} f(S(t+\tau), I(t))-\frac{K\left(S_{0}\right)}{K(S(t))} f(S(t), I(t-\tau))\right\} d \tau \\
= & \left(1-\frac{K\left(S_{0}\right)}{K(S(t))}\right)\left(-\mu\left(S(t)-S_{0}\right)+\delta R(t)\right) \\
& +\int_{0}^{h} p(\tau)\left\{\frac{K\left(S_{0}\right)}{K(S(t+\tau))} \frac{f(S(t+\tau), I(t))}{I(t)}-(\mu+\gamma)\right\} I(t) d \tau .
\end{aligned}
$$

Noting that $\delta R(t)\left(1-\frac{K\left(S_{0}\right)}{K(S(t))}\right) \leq 0$ on the plane $\left\{(S, I, R) \in \mathbb{R}_{+}^{3} \mid S+I+R=S_{0}\right\}$, we obtain

$$
\begin{aligned}
\frac{\mathrm{d} W(t)}{\mathrm{d} t} \leq & -\mu\left(1-\frac{K\left(S_{0}\right)}{K(S(t))}\right)\left(S(t)-S_{0}\right) \\
& +\int_{0}^{h} p(\tau)\left\{\frac{K\left(S_{0}\right)}{K(S(t+\tau))} K(S(t+\tau))-(\mu+\gamma)\right\} I(t) d \tau \\
= & -\mu\left(1-\frac{K\left(S_{0}\right)}{K(S(t))}\right)\left(S(t)-S_{0}\right)+(\mu+\gamma)\left(R_{0}-1\right) I(t)
\end{aligned}
$$

Thus, it holds that $\frac{\mathrm{d} W(t)}{\mathrm{d} t} \leq 0$ with equality if $S(t)=S_{0}$. Hence, we have $\lim _{t \rightarrow+\infty} S(t)=S_{0}$, which implies from system (3.1) that $\lim _{t \rightarrow+\infty} I(t)=0$ and $\lim _{t \rightarrow+\infty} R(t)=0$ hold. By an extension of Lyapunov-LaSalle asymptotic stability theorem (see also Kuang [13, Theorem 5.3]), the disease-free equilibrium $E_{0}$ of system (3.1) is globally asymptotically stable. This completes the proof.

Proof of Theorem 1.1. From Theorem 3.1, we immediately obtain the conclusion of this theorem.

\section{Global stability of the endemic equilibrium $E_{*}$ for $R_{0}>1$}

In this section, we establish the global asymptotic stability of the endemic equilibrium $E_{*}$ of system (1.2) for $R_{0}>1$ by using a lower bound of the susceptible individuals $S(t)$ for large $t$.

First, we obtain the following theorem, which indicates that the disease eventually persists in the host population when $R_{0}>1$. 
Theorem 4.1 If $R_{0}>1$, then for any solution of system (1.2), it holds that

$$
\left\{\begin{array}{l}
\liminf _{t \rightarrow+\infty} S(t) \geq v_{1}, \liminf _{t \rightarrow+\infty} I(t) \geq v_{2}:=q I^{*} \exp (-(\mu+\gamma) \rho h), \\
\liminf _{t \rightarrow+\infty} R(t) \geq v_{3}:=\frac{\gamma v_{2}}{\mu+\delta}
\end{array}\right.
$$

where $v_{1}>0$ satisfies $\mu-K\left(v_{1}\right)-\mu v_{1}=0$ and $0<q<1$ and $\rho \geq 1$ satisfy

$$
S^{*}<\frac{\mu-\left(K\left(S_{0}\right)+\varepsilon_{S}\right) q I^{*}}{\mu}\left(1-\mathrm{e}^{-\mu \rho h}\right), 0<q<\frac{\mu}{\left(K\left(S_{0}\right)+\varepsilon_{S}\right) I^{*}} .
$$

Proof Let $(S(t), I(t), R(t))$ be a solution of system (1.2) with initial condition (1.3). By Lemma 2.1, it follows that $\limsup _{t \rightarrow+\infty} I(t) \leq 1$, which implies from the first equation of system (1.2) and the hypothesis (H2) that, for any $\varepsilon_{I}>0$, there is an integer $T_{I} \geq 0$ such that

$$
\begin{aligned}
\frac{\mathrm{d} S(t)}{\mathrm{d} t} & \geq \mu-\int_{0}^{h} p(\tau) \frac{f(S(t), I(t-\tau))}{I(t-\tau)} I(t-\tau) d \tau-\mu S(t) \\
& \geq \mu-K(S(t)) \int_{0}^{h} p(\tau) I(t-\tau) d \tau-\mu S(t) \\
& =\mu-K(S(t))\left(1+\varepsilon_{I}\right)-\mu S(t)
\end{aligned}
$$

for $t \geq T_{I}+h$. Let us now consider the following auxiliary equation:

$$
\frac{\mathrm{d} S(t)}{\mathrm{d} t}=\mu-K(S(t))-\mu S(t)
$$

Then one can obtain that $\lim _{t \rightarrow+\infty} S(t)=v_{1}>0$. Since (4.2) holds for arbitrary $\varepsilon_{I}>0$ sufficiently small, it follows that $\liminf _{t \rightarrow+\infty} S(t) \geq v_{1}>0$.

We now prove that it is impossible that $I(t) \leq q I^{*}$ for all sufficiently large $t$. Suppose to the contrary that there exists a sufficiently large $t_{1} \geq T_{S}$ such that $I(t) \leq q I^{*}$ holds for all $t \geq t_{1}$. Then, similar to the above discussion, we have that for any $t \geq t_{1}+h$,

$$
\begin{aligned}
\frac{\mathrm{d} S(t)}{\mathrm{d} t} & \geq \mu-\int_{0}^{h} p(\tau) \phi(S(t), I(t-\tau)) I(t-\tau) d \tau-\mu S(t) \\
& \geq \mu-\left(K\left(S_{0}\right)+\varepsilon_{S}\right) q I^{*}-\mu S(t)
\end{aligned}
$$

which yields for $t \geq t_{1}+h$,

$$
\begin{aligned}
S(t) & \geq S\left(t_{1}+h\right) \mathrm{e}^{-\mu\left(t-t_{1}-h\right)}+\mathrm{e}^{-\mu t} \int_{t_{1}+h}^{t} \mathrm{e}^{\mu s}\left(\mu-\left(K\left(S_{0}\right)+\varepsilon_{S}\right) q I^{*}\right) d s \\
& =S\left(t_{1}+h\right) \mathrm{e}^{-\mu\left(t-t_{1}-h\right)}+\frac{\mu-\left(K\left(S_{0}\right)+\varepsilon_{S}\right) q I^{*}}{\mu}\left(1-\mathrm{e}^{-\mu\left(t-t_{1}-h\right)}\right) .
\end{aligned}
$$

Hence, it follows from (4.3) that for $t \geq t_{1}+h+\rho h$,

$$
\begin{aligned}
S(t) & >\frac{\mu-\left(K\left(S_{0}\right)+\varepsilon_{S}\right) q I^{*}}{\mu}\left(1-\mathrm{e}^{-\mu \rho h}\right) \\
& =S^{\triangle}>S^{*} .
\end{aligned}
$$

Now, we define the following function:

$$
V(t)=I(t)+\int_{0}^{h} p(\tau) \int_{t}^{t+\tau} f(S(u), I(u-\tau)) d u d \tau .
$$


Calculating the derivative of $V(t)$ along solutions of system (1.2) gives as follows:

$$
\begin{aligned}
\frac{\mathrm{d} V(t)}{\mathrm{d} t}= & \int_{0}^{h} p(\tau) f(S(t), I(t-\tau)) d \tau-(\mu+\gamma) I(t) \\
& +\int_{0}^{h} p(\tau)\{f(S(t+\tau), I(t))-f(S(t), I(t-\tau))\} d \tau \\
= & \int_{0}^{h} p(\tau) f(S(t+\tau), I(t)) d \tau-(\mu+\gamma) I(t)
\end{aligned}
$$

For $t \geq t_{1}+h+\rho h$, it follows from (4.4) and the relation $\mu+\gamma=\phi\left(S^{*}, I^{*}\right)$ that

$$
\begin{aligned}
\frac{\mathrm{d} V(t)}{\mathrm{d} t} & =\int_{0}^{h} p(\tau)\{\phi(S(t+\tau), I(t))-(\mu+\gamma)\} I(t) d \tau \\
& >\int_{0}^{h} p(\tau)\left\{\phi\left(S(t+\tau), I^{*}\right)-\phi\left(S^{*}, I^{*}\right)+\phi\left(S^{*}, I^{*}\right)-(\mu+\gamma)\right\} I(t) d \tau \\
& =\int_{0}^{h} p(\tau)\left\{\phi\left(S(t+\tau), I^{*}\right)-\phi\left(S^{*}, I^{*}\right)\right\} I(t) d \tau \\
& \geq\left\{\phi\left(S^{\triangle}, I^{*}\right)-\phi\left(S^{*}, I^{*}\right)\right\} I(t) .
\end{aligned}
$$

Setting $\underline{i}=\min _{\theta \in[-h, 0]} I\left(\theta+t_{1}+\rho h+2 h\right)$, we claim that $I(t) \geq \underline{i}$ for all $t \geq t_{1}+h+\rho h$. Otherwise, if there is a $T \geq 0$ such that $I(t) \geq \underline{i}$ for $t_{1}+h+\rho h \leq t \leq t_{1}+2 h+\rho h+T$, $I\left(t_{1}+2 h+\rho h+T\right)=\underline{i}$ and $\left.\frac{\mathrm{d} I(t)}{\mathrm{d} t}\right|_{t=t_{1}+2 h+\rho h+T} \leq 0$, it follows from the second equation of system (1.2), the conditions (H1) and (H2) that for $t_{2}=t_{1}+2 h+\rho h+T$,

$$
\begin{aligned}
\left.\frac{\mathrm{d} I(t)}{\mathrm{d} t}\right|_{t=t_{2}} & =\int_{0}^{h} p(\tau) f\left(S\left(t_{2}\right), I\left(t_{2}-\tau\right)\right) d \tau-(\mu+\gamma) I\left(t_{2}\right) \\
& =\int_{0}^{h} p(\tau) \phi\left(S\left(t_{2}\right), I\left(t_{2}-\tau\right)\right) I\left(t_{2}-\tau\right) d \tau-(\mu+\gamma) I\left(t_{2}\right) \\
& >\int_{0}^{h} p(\tau) \phi\left(S\left(t_{2}\right), I^{*}\right) I\left(t_{2}-\tau\right) d \tau-(\mu+\gamma) I\left(t_{2}\right) \\
& \geq\left\{\phi\left(S\left(t_{2}\right), I^{*}\right)-(\mu+\gamma)\right\} I\left(t_{2}\right) \\
& \geq\left\{\phi\left(S^{\triangle}, I^{*}\right)-(\mu+\gamma)\right\} \underline{i} \\
& >\left\{\phi\left(S^{*}, I^{*}\right)-(\mu+\gamma)\right\} \underline{i}=0 .
\end{aligned}
$$

This is a contradiction. Therefore $I(t) \geq \underline{i}$ for all $t \geq t_{1}+h+\rho h$. It follows from (4.6) that

$$
\frac{d V(t)}{d t}>\left\{\phi\left(S^{\triangle}, I^{*}\right)-\phi\left(S^{*}, I^{*}\right)\right\} \underline{i}>0, \text { for } t \geq t_{1}+2 h+\rho h,
$$

which implies that $\lim _{t \rightarrow+\infty} V(t)=+\infty$. However, it holds from (2.1) and (4.5) that $\limsup _{t \rightarrow+\infty} V(t)<$ $+\infty$. Hence the claim holds.

Thus, we proved that it is impossible that $I(t) \leq q I^{*}$ for all sufficiently large $t$. Now, we are left to consider the following two possibilities:

$\left\{\left(\right.\right.$ i) $I(t) \geq q I^{*}$ for all $t$ sufficiently large,

(ii) $I(t)$ oscillates about $q I^{*}$ for all $t$ sufficiently large. 
If the first case holds, then we immediately get the conclusion of the proof. If the second case holds, we show that $I(t) \geq q I^{*} \exp (-(\mu+\gamma) \rho h)$ for all $t$ sufficiently large. Let $t_{3}<t_{4}$ be sufficiently large such that

$$
I\left(t_{3}\right)=I\left(t_{4}\right)=q I^{*}, I(t)<q I^{*}, t_{3}<t<t_{4} .
$$

If $t_{4}-t_{3} \leq \rho h$, then it follows from the second equation of system (1.2) that

$$
\frac{\mathrm{d} I(t)}{\mathrm{d} t}>-(\mu+\gamma) I(t)
$$

that is,

$$
\begin{aligned}
I(t) & >I\left(t_{3}\right) \exp \left(-(\mu+\gamma)\left(t-t_{3}\right)\right) \\
& \geq q I^{*} \exp (-(\mu+\gamma) \rho h)=v_{2} .
\end{aligned}
$$

If $t_{4}-t_{3}>\rho h$, we obtain from the second equation of system (1.2) that $I(t) \geq v_{2}$ for $t_{3} \leq t \leq$ $t_{3}+\rho h$. We now claim that $I(t) \geq v_{2}$ for all $t_{3}+\rho h \leq t \leq t_{4}$. Otherwise, there is a $T^{*}>0$ such that $I(t) \geq v_{2}$ for $t_{3} \leq t \leq t_{3}+\rho h+T^{*}<t_{4}, I\left(t_{3}+\rho h+T^{*}\right)=v_{2}$ and $\left.\frac{\mathrm{d} I(t)}{\mathrm{d} t}\right|_{t=t_{3}+\rho h+T^{*}} \leq 0$. On the other hand, for $t_{0}=t_{3}+\rho h+T^{*}$, it follows from the second equation of system (1.2) and the relation $\phi\left(S\left(t_{0}\right), I\left(t_{0}\right)\right)>\phi\left(S\left(t_{0}\right), I^{*}\right) \geq \phi\left(S^{\triangle}, I^{*}\right)>\phi\left(S^{*}, I^{*}\right)$ that

$$
\begin{aligned}
\left.\frac{\mathrm{d} I(t)}{\mathrm{d} t}\right|_{t=t_{0}} & =\int_{0}^{h} p(\tau) f\left(S\left(t_{0}\right), I\left(t_{0}-\tau\right)\right) d \tau-(\mu+\gamma) I\left(t_{0}\right) \\
& =\int_{0}^{h} p(\tau) \phi\left(S\left(t_{0}\right), I\left(t_{0}-\tau\right)\right) I\left(t_{0}-\tau\right) d \tau-(\mu+\gamma) I\left(t_{0}\right) \\
& >\left\{\phi\left(S\left(t_{0}\right), I^{*}\right)-(\mu+\gamma)\right\} I\left(t_{0}\right) \\
& \geq\left\{\phi\left(S^{\triangle}, I^{*}\right)-(\mu+\gamma)\right\} I\left(t_{0}\right) \\
& >\left\{\phi\left(S^{*}, I^{*}\right)-(\mu+\gamma)\right\} I\left(t_{0}\right)=0,
\end{aligned}
$$

which is a contradiction. Hence $I(t) \geq q I^{*} \exp (-(\mu+\gamma) \rho h)=v_{2}$ for $t_{3} \leq t \leq t_{4}$. Since the interval $\left[t_{3}, t_{4}\right]$ is arbitrarily chosen, we conclude that $I(t) \geq v_{2}$ for all $t$ sufficiently large for the second case. Thus, we obtain that $\liminf _{t \rightarrow+\infty} I(t) \geq v_{2}$. From the above discussion, one can see that $\liminf _{t \rightarrow+\infty} R(t) \geq v_{3}$. Hence, this completes the proof.

Recalling by Lemma 2.2 that the existence of the endemic equilibrium $\tilde{E}_{*} \equiv\left(S^{*}, I^{*}\right)$ of system (4.10) is guaranteed for the case $R_{0}>1$, for a fixed $0 \leq \tau \leq h$, we put

$$
\left\{\begin{array}{l}
x_{t}=\frac{S(t)}{S^{*}}, \tilde{x}_{t}=\frac{f\left(S(t), I^{*}\right)}{f\left(S^{*}, I^{*}\right)}, y_{t}=\frac{I(t)}{I^{*}}, \tilde{y}_{t, \tau}=\frac{f(S(t+\tau), I(t))}{f\left(S(t+\tau), I^{*}\right)} \\
g(x)=x-1-\ln x \geq g(1)=0, \text { for } x>0
\end{array}\right.
$$

The following lemma plays a key role to obtain Theorem 1.2.

Lemma 4.1 For all $t \geq 0$ and $0 \leq \tau \leq h$, it holds that

$$
\left(1-x_{t}\right)\left(1-\frac{1}{\tilde{x}_{t}}\right) \leq-C_{0} \frac{f\left(S(t), I^{*}\right)}{S^{*} I^{*}}\left(1-\frac{1}{\tilde{x}_{t}}\right)^{2}
$$

and

$$
g\left(y_{t}\right)-g\left(\tilde{y}_{t, \tau}\right) \geq C_{1} C_{2} I^{*}\left(y_{t}-1\right)^{2},
$$

with equality if and only if $x_{t}=1$ and $y_{t}=\tilde{y}_{t, \tau}=1$, respectively. 
Proof First, by the condition (I) of (1.5), we obtain that

$$
\begin{aligned}
\left(1-x_{t}\right)\left(1-\frac{1}{\tilde{x}_{t}}\right) & =\left(1-\frac{S(t)}{S^{*}}\right)\left(1-\frac{f\left(S^{*}, I^{*}\right)}{f\left(S(t), I^{*}\right)}\right) \\
& =-\frac{1}{S^{*}}\left(S(t)-S^{*}\right)\left(1-\frac{f\left(S^{*}, I^{*}\right)}{f\left(S(t), I^{*}\right)}\right) \\
& \leq-\frac{C_{0}}{S^{*} I^{*}}\left(f\left(S(t), I^{*}\right)-f\left(S^{*}, I^{*}\right)\right)\left(1-\frac{f\left(S^{*}, I^{*}\right)}{f\left(S(t), I^{*}\right)}\right) \\
& =-C_{0} \frac{f\left(S(t), I^{*}\right)}{S^{*} I^{*}}\left(1-\frac{1}{\tilde{x}_{t}}\right)^{2} .
\end{aligned}
$$

Second, we have that

$$
\tilde{y}_{t, \tau}-1=\frac{f(S(t+\tau), I(t))-f\left(S(t+\tau), I^{*}\right)}{f\left(S(t+\tau), I^{*}\right)}
$$

and

$$
\begin{aligned}
y_{t}-\tilde{y}_{t, \tau} & =\frac{I(t)}{I^{*}}-\frac{f(S(t+\tau), I(t))}{f\left(S(t+\tau), I^{*}\right)} \\
& =\frac{f(S(t+\tau), I(t))}{I^{*}}\left(\frac{I(t)}{f(S(t+\tau), I(t))}-\frac{I^{*}}{f\left(S(t+\tau), I^{*}\right)}\right) .
\end{aligned}
$$

Then, by the hypotheses (H1) and (H2), we immediately obtain

$$
\begin{aligned}
\left(y_{t}-\tilde{y}_{t, \tau}\right)\left(\tilde{y}_{t, \tau}-1\right)= & \frac{f(S(t+\tau), I(t))}{I^{*} f\left(S(t+\tau), I^{*}\right)}\left(\frac{I(t)}{f(S(t+\tau), I(t))}-\frac{I^{*}}{f\left(S(t+\tau), I^{*}\right)}\right) \\
& \times\left(f(S(t+\tau), I(t))-f\left(S(t+\tau), I^{*}\right)\right) \\
\geq & 0,
\end{aligned}
$$

with equality if and only if $y_{t}=\tilde{y}_{t, \tau}=1$. Moreover, since $g^{\prime}(x)=1-\frac{1}{x}$ and $g^{\prime \prime}(x)=\frac{1}{x^{2}} \geq 0$ for all $x>0$, it holds that

$$
\begin{aligned}
g\left(y_{t}\right)-g\left(\tilde{y}_{t, \tau}\right) \geq & g^{\prime}\left(\tilde{y}_{t, \tau}\right)\left(y_{t}-\tilde{y}_{t, \tau}\right) \\
\geq & \frac{\tilde{y}_{t, \tau}-1}{\tilde{y}_{t, \tau}}\left(y_{t}-\tilde{y}_{t, \tau}\right) \\
= & \frac{1}{I^{*}}\left(\frac{I(t)}{f(S(t+\tau), I(t))}-\frac{I^{*}}{f\left(S(t+\tau), I^{*}\right)}\right) \\
& \times\left(f(S(t+\tau), I(t))-f\left(S(t+\tau), I^{*}\right)\right) \\
\geq & \frac{C_{1} C_{2}}{I^{*}}\left(I(t)-I^{*}\right)^{2} \\
= & C_{1} C_{2} I^{*}\left(y_{t}-1\right)^{2},
\end{aligned}
$$

with equality if and only if $y_{t}=\tilde{y}_{t, \tau}=1$. Hence, we get the conclusion. 
Now, we are in a position to prove the global asymptotic stability of the endemic equilibrium $E_{*}$ of system (1.2) for $R_{0}>1$.

Proof of Theorem 1.2. From Lemma 2.1, the limit set of system (1.2) in the first octant of $\mathbb{R}^{3}$ locates on the plane $S+I+R=S_{0}$. Hence, the dynamics of system (1.2) in the first octant of $\mathbb{R}^{3}$ is equivalent to the following system:

$$
\left\{\begin{array}{l}
\frac{\mathrm{d} S(t)}{\mathrm{d} t}=(\mu+\delta)-(\mu+\delta) S(t)-\int_{0}^{h} p(\tau) f(S(t), I(t-\tau)) d \tau-\delta I(t) \\
\frac{\mathrm{d} I(t)}{\mathrm{d} t}=\int_{0}^{h} p(\tau) f(S(t), I(t-\tau)) d \tau-(\mu+\gamma) I(t)
\end{array}\right.
$$

We consider the following Lyapunov functional (cf. $[5,7,14,15])$ :

$$
V(t)=U(t)+U_{+}(t)
$$

where

$$
\left\{\begin{array}{l}
U(t)=\int_{S^{*}}^{S(t)}\left(1-\frac{f\left(S^{*}, I^{*}\right)}{f\left(\tau, I^{*}\right)}\right) d \tau+\left(I(t)-I^{*}-\ln \frac{I(t)}{I^{*}}\right), \\
U_{+}(t)=f\left(S^{*}, I^{*}\right) \int_{0}^{h} p(\tau) \int_{t-\tau}^{t} g\left(\frac{f(S(u+\tau), I(u)}{f\left(S(u+\tau), I^{*}\right)}\right) d u d \tau .
\end{array}\right.
$$

The time derivative of $\frac{\mathrm{d} U(t)}{\mathrm{d} t}$ along the solution of system (4.10) satisfies as follows:

$$
\begin{aligned}
\frac{\mathrm{d} U(t)}{\mathrm{d} t}= & \left(1-\frac{f\left(S^{*}, I^{*}\right)}{f\left(S(t), I^{*}\right)}\right)\left\{(\mu+\delta)-\int_{0}^{h} p(\tau) f(S(t), I(t-\tau)) d \tau-(\mu+\delta) S(t)-\delta I(t)\right\} \\
& +\left(1-\frac{I^{*}}{I(t)}\right)\left(\int_{0}^{h} p(\tau) f(S(t), I(t-\tau)) d \tau-(\mu+\gamma) I(t)\right)
\end{aligned}
$$


Rearranging $\mu+\delta=(\mu+\delta) S^{*}+f\left(S^{*}, I^{*}\right)+\delta I^{*}$ and $\mu+\gamma=\frac{f\left(S^{*} I^{*}\right)}{I^{*}}$ gives

$$
\begin{aligned}
& \frac{\mathrm{d} U(t)}{\mathrm{d} t}=\left(1-\frac{f\left(S^{*}, I^{*}\right)}{f\left(S(t), I^{*}\right)}\right) \\
& \times\left\{(\mu+\delta)\left(S^{*}-S(t)\right)+\left(f\left(S^{*}, I^{*}\right)-\int_{0}^{h} p(\tau) f(S(t), I(t-\tau)) d \tau\right)\right\} \\
& +\left(1-\frac{I^{*}}{I(t)}\right)\left(\int_{0}^{h} p(\tau) f(S(t), I(t-\tau)) d \tau-f\left(S^{*}, I^{*}\right) \frac{I(t)}{I^{*}}\right) \\
& +\delta\left(1-\frac{f\left(S^{*}, I^{*}\right)}{f\left(S(t), I^{*}\right)}\right)\left(I^{*}-I(t)\right) \\
& =(\mu+\delta) S^{*}\left(1-\frac{S(t)}{S^{*}}\right)\left(1-\frac{f\left(S^{*}, I^{*}\right)}{f\left(S(t), I^{*}\right)}\right) \\
& +f\left(S^{*}, I^{*}\right)\left(1-\frac{f\left(S^{*}, I^{*}\right)}{f\left(S(t), I^{*}\right)}\right) \int_{0}^{h} p(\tau)\left(1-\frac{f(S(t), I(t-\tau))}{f\left(S^{*}, I^{*}\right)}\right) d \tau \\
& +f\left(S^{*}, I^{*}\right)\left(1-\frac{I^{*}}{I(t)}\right) \int_{0}^{h} p(\tau)\left(\frac{f(S(t), I(t-\tau))}{f\left(S^{*}, I^{*}\right)}-\frac{I(t)}{I^{*}}\right) d \tau \\
& +\delta\left(1-\frac{f\left(S^{*}, I^{*}\right)}{f\left(S(t), I^{*}\right)}\right)\left(I^{*}-I(t)\right) \\
& =(\mu+\delta) S^{*}\left(1-\frac{S(t)}{S^{*}}\right)\left(1-\frac{f\left(S^{*}, I^{*}\right)}{f\left(S(t), I^{*}\right)}\right) \\
& +f\left(S^{*}, I^{*}\right) \int_{0}^{h} p(\tau)\left\{\left(2-\frac{f\left(S^{*}, I^{*}\right)}{f\left(S(t), I^{*}\right)}-\frac{I^{*}}{I(t)} \frac{f(S(t), I(t-\tau))}{f\left(S^{*}, I^{*}\right)}\right)\right. \\
& \left.+\left(\frac{f(S(t), I(t-\tau))}{f\left(S(t), I^{*}\right)}-\frac{I(t)}{I^{*}}\right)\right\} d \tau+\delta\left(1-\frac{f\left(S^{*}, I^{*}\right)}{f\left(S(t), I^{*}\right)}\right)\left(I^{*}-I(t)\right) \\
& =(\mu+\delta) S^{*}\left(1-\frac{S(t)}{S^{*}}\right)\left(1-\frac{f\left(S^{*}, I^{*}\right)}{f\left(S(t), I^{*}\right)}\right) \\
& +f\left(S^{*}, I^{*}\right) \int_{0}^{h} p(\tau)\left\{-g\left(\frac{f\left(S^{*}, I^{*}\right)}{f\left(S(t), I^{*}\right)}\right)-g\left(\frac{I^{*}}{I(t)} \frac{f(S(t), I(t-\tau))}{f\left(S^{*}, I^{*}\right)}\right)\right. \\
& \left.+g\left(\frac{f(S(t), I(t-\tau))}{f\left(S(t), I^{*}\right)}\right)-g\left(\frac{I(t)}{I^{*}}\right)\right\} d \tau+\delta\left(1-\frac{f\left(S^{*}, I^{*}\right)}{f\left(S(t), I^{*}\right)}\right)\left(I^{*}-I(t)\right) .
\end{aligned}
$$

Second, calculating $\frac{\mathrm{d} U_{+}(t)}{\mathrm{d} t}$ gives as follows.

$$
\frac{\mathrm{d} U_{+}(t)}{\mathrm{d} t}=f\left(S^{*}, I^{*}\right) \int_{0}^{h} p(\tau)\left\{g\left(\frac{f(S(t+\tau), I(t))}{f\left(S(t+\tau), I^{*}\right)}\right)-g\left(\frac{f(S(t), I(t-\tau))}{f\left(S(t), I^{*}\right)}\right)\right\} d \tau .
$$


Finally, we obtain that

$$
\begin{aligned}
\frac{\mathrm{d} V(t)}{\mathrm{d} t}= & (\mu+\delta) S^{*}\left(1-\frac{S(t)}{S^{*}}\right)\left(1-\frac{f\left(S^{*}, I^{*}\right)}{f\left(S(t), I^{*}\right)}\right) \\
& +f\left(S^{*}, I^{*}\right) \int_{0}^{h} p(\tau)\left\{-g\left(\frac{f\left(S^{*}, I^{*}\right)}{f\left(S(t), I^{*}\right)}\right)-g\left(\frac{I^{*}}{I(t)} \frac{f(S(t), I(t-\tau))}{f\left(S^{*}, I^{*}\right)}\right)\right. \\
& \left.+g\left(\frac{f(S(t+\tau), I(t))}{f\left(S(t+\tau), I^{*}\right)}\right)-g\left(\frac{I(t)}{I^{*}}\right)\right\} d \tau \\
& +\delta\left(1-\frac{f\left(S^{*}, I^{*}\right)}{f\left(S(t), I^{*}\right)}\right)\left(I^{*}-I(t)\right) \\
= & (\mu+\delta) S^{*}\left(1-x_{t}\right)\left(1-\frac{1}{\tilde{x}_{t}}\right)-\delta I^{*}\left(1-\frac{1}{\tilde{x}_{t}}\right)\left(y_{t}-1\right) \\
& -f\left(S^{*}, I^{*}\right) \int_{0}^{h} p(\tau)\left\{g\left(\frac{1}{\tilde{x}_{t}}\right)+g\left(\frac{\tilde{x}_{t} \tilde{y}_{t-\tau, \tau}}{y_{t}}\right)+g\left(y_{t}\right)-g\left(\tilde{y}_{t, \tau}\right)\right\} d \tau .
\end{aligned}
$$

By applying (4.8), (4.9) and the relation $g\left(\frac{1}{\tilde{x}_{t}}\right)+g\left(\frac{\tilde{x}_{t} \tilde{y}_{t-\tau, \tau}}{y_{t}}\right) \geq 0$ to (4.12), it holds that

$$
\begin{aligned}
\frac{\mathrm{d} V(t)}{\mathrm{d} t} \leq & -C_{0} \frac{f\left(S(t), I^{*}\right)}{I^{*}}(\mu+\delta)\left(1-\frac{1}{\tilde{x}_{t}}\right)^{2} \\
& -\delta I^{*}\left(1-\frac{1}{\tilde{x}_{t}}\right)\left(y_{t}-1\right)-C_{1} C_{2} f\left(S^{*}, I^{*}\right) I^{*}\left(y_{t}-1\right)^{2}
\end{aligned}
$$

Using $f\left(S^{*}, I^{*}\right)=(\mu+\gamma) I^{*}$ gives as follows. we have that

$$
\begin{aligned}
\frac{\mathrm{d} V(t)}{\mathrm{d} t} \leq & -\left\{C_{0} \frac{f\left(S(t), I^{*}\right)}{I^{*}}(\mu+\delta)\left(1-\frac{1}{\tilde{x}_{t}}\right)^{2}+\delta I^{*}\left(1-\frac{1}{\tilde{x}_{t}}\right)\left(y_{t}-1\right)\right. \\
& \left.+C_{1} C_{2}(\mu+\gamma)\left(I^{*}\right)^{2}\left(y_{t}-1\right)^{2}\right\}
\end{aligned}
$$

By Theorem 4.1, for any $0<\varepsilon<v_{1}$, there exists a $T_{\varepsilon}>0$ such that $S(t)>v_{1}-\varepsilon$ for any $t>T_{\varepsilon}$. From the condition (II) of (1.5), we may restrict this $\varepsilon>0$ sufficiently small such that

$$
\delta^{2}-4 C_{0} C_{1} C_{2}(\mu+\delta)(\mu+\gamma) \frac{f\left(v_{1}-\varepsilon, I^{*}\right)}{I^{*}}<0
$$

Then, we have that

$$
\begin{aligned}
& \left(\delta I^{*}\right)^{2}-4\left\{C_{0} \frac{f\left(S(t), I^{*}\right)}{I^{*}}(\mu+\delta)\right\}\left\{C_{1} C_{2}(\mu+\gamma)\left(I^{*}\right)^{2}\right\} \\
< & \left(I^{*}\right)^{2}\left\{\delta^{2}-4 C_{0} C_{1} C_{2}(\mu+\delta)(\mu+\gamma) \frac{f\left(v_{1}-\varepsilon, I^{*}\right)}{I^{*}}\right\}<0,
\end{aligned}
$$

from which we obtain that $\frac{\mathrm{d} V(t)}{\mathrm{d} t} \leq 0$ holds for all $t>T_{\varepsilon}$ with equality if and only if $S(t)=S^{*}$ and $I(t)=I^{*}$. Thus, by an extention of LaSalle's invariant principle (see also Kuang [13, Corollary 5.2]), $E_{*}$ is globally asymptotically stable. Hence, the proof is complete. 


\section{Applications}

In this section, we illustrate some examples in order to validate the feasibility of our global stability results with respect to the rate of immunity lost $\delta$. We consider the following SIRS epideimc model with a discrete delay:

$$
\left\{\begin{array}{l}
\frac{\mathrm{d} S(t)}{\mathrm{d} t}=\mu-\mu S(t)-\beta \frac{S(t)}{1+\alpha_{S} S(t)} \frac{I(t-\tau)}{1+\alpha_{I} I(t-\tau)}+\delta R(t), \\
\frac{\mathrm{d} I(t)}{\mathrm{d} t}=\beta \frac{S(t)}{1+\alpha_{S} S(t)} \frac{I(t-\tau)}{1+\alpha_{I} I(t-\tau)}-(\mu+\gamma) I(t), \\
\frac{\mathrm{d} R(t)}{\mathrm{d} t}=\gamma I(t)-(\mu+\delta) R(t), \tau>0 .
\end{array}\right.
$$

$\beta>0$ denotes the infection force of disease. Here, $\frac{1}{1+\alpha_{S} S(t)}$ and $\frac{1}{1+\alpha_{I} I(t-\tau)}$ measures the inhibition effects from the behavioral change of the susceptible individuals and infective individuals, respectively. For the case $\alpha_{S}=\alpha_{I}=0$, the incidence rate becomes a form which is proposed in Vargas-De-León and Gómez-Alcaraz [22] and for the case $\alpha_{S}=0$, the incidence rate becomes a form which is proposed in Xu and Ma [23]. From (1.4), the basic reproduction number of system (5.1) becomes $R_{0}=\frac{\beta}{(\mu+\gamma)\left(1+\alpha_{S}\right)}$.

Using similar techniques in the proofs of Theorems 1.1 and 1.2, we establish the following result (cf. McCluskey [14, Section 5]):

Corollary 5.1 If $R_{0} \leq 1$, then the disease-free equilibrium $E_{0}$ of system (1.2) is globally asymptotically stable. If $R_{0}>1$, then the endemic equilibrium $E_{*}$ of system (5.1) exists uniquely and system (5.1) is permanent. Moreover, the endemic equilibrium $E_{*}$ of system (5.1) is globally asymptotically stable if

$$
\left\{\begin{array}{l}
\delta^{2}<4 \tilde{C}_{0} \tilde{C}_{1} \tilde{C}_{2}(\mu+\delta)(\mu+\gamma) \frac{\beta \tilde{v}_{1}}{\left(1+\alpha_{S} \tilde{v}_{1}\right)\left(1+\alpha_{I} I^{*}\right)} \\
\tilde{C}_{0}=\frac{\left(1+\alpha_{S} \tilde{v}_{1}\right)\left(1+\alpha_{I} I^{*}\right)}{\beta} \leq \frac{\left(1+\alpha_{S} S^{*}\right)\left(1+\alpha_{I} I^{*}\right)}{\beta} \\
\tilde{C}_{1}=\frac{\beta}{\left(1+\alpha_{S} \tilde{v}_{1}\right)\left(1+\alpha_{I}\right)^{2}} \leq \frac{\beta}{\left(1+\alpha_{S} \tilde{v}_{1}\right)\left(1+\alpha_{I}\right)\left(1+\alpha_{I} I^{*}\right)} \\
\tilde{C}_{2}=\frac{\left(1+\alpha_{S}\right) \alpha_{I}}{\beta}
\end{array}\right.
$$

where $v=\tilde{v}_{1}$ is a unique positive solution of $\mu-\frac{\beta v}{\left(1+\alpha_{S} v\right)}-\mu v=0$.

For system (5.1), under the conditions

$$
\tau=0.1, \alpha_{S}=\alpha_{I}=0.1, \gamma=0.01 \text { and } \mu=0.02,
$$

we consider two cases of $\beta=0.02$ and $\beta=0.06$. First, we consider the case $\beta=0.02$. Then, we obtain $R_{0}=0.606 \cdots \leq 1$. By Theorem 1.1 , the disease-free equilibrium $E_{0}$ of system (5.1) is globally asymptotically stable for any $\delta \geq 0$.

Second, we consider the case $\beta=0.06$. Then, we obtain $R_{0}=1.818 \cdots>1$ and $\tilde{v}_{1}=$ $0.512 \cdots$. For this case, since the condition (5.2) becomes $0<\delta<\delta^{*}:=0.013 \cdots$, the endemic equilibrium $E_{*}$ of system (5.1) is globally asymptotically stable for any $0<\delta<\delta^{*}$. From a biological point of view, for a small loss of immunity rate, the prevalence of the disease can settle to an endemic steady state independently of the initial conditions. 


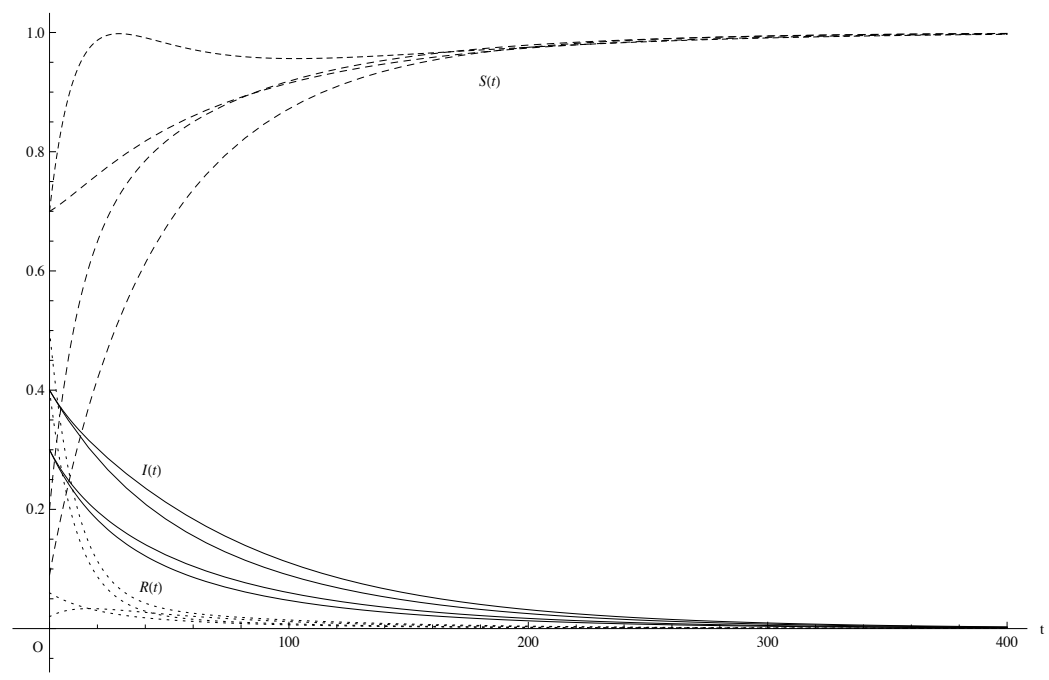

Fig. 1 The graph trajectory of $S(t), I(t)$ and $R(t)$ of system (5.1). For the case (5.3) with $\beta=0.02$ and $\delta=0.07$, we have $R_{0}=0.606 \cdots<1$ and $E_{0}=(1,0,0)$.

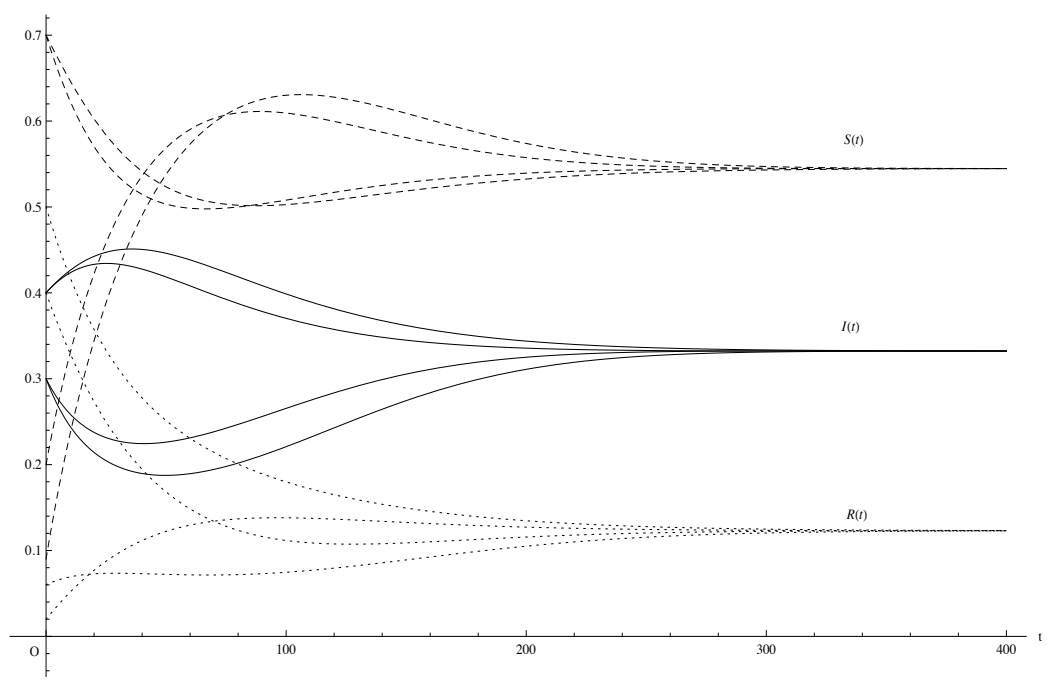

Fig. 2 The graph trajectory of $S(t), I(t)$ and $R(t)$ of system (5.1). For the case (5.3) with $\beta=0.06$ and $\delta=0.007<\delta^{*}$, we have $R_{0}=1.818 \cdots>1$ and $E^{*}=(0.544 \cdots, 0.332 \cdots, 0.123 \cdots)$.

Figures 1 and 2 indicate that the disease-free equilibrium $E_{0}$ and the endemic equilibrium $E_{*}$ of system (5.1) are globally asymptotically stable for the first and the second cases with $\delta=0.07$ and $\delta=0.007$, respectively.

On the other hand, Figure 3 indicates that the endemic equilibrium $E_{*}$ of system (5.1) is also globally asymptotically stable even if the condition (5.2) in Corollary 5.1 fail for the second case with $\delta=0.07 \geq \delta^{*}$ for $R_{0}>1$. There is still an open problem to determine the global asymptotic stability of the endemic equilibrium of system (5.1) for $\delta \geq \delta^{*}$. 


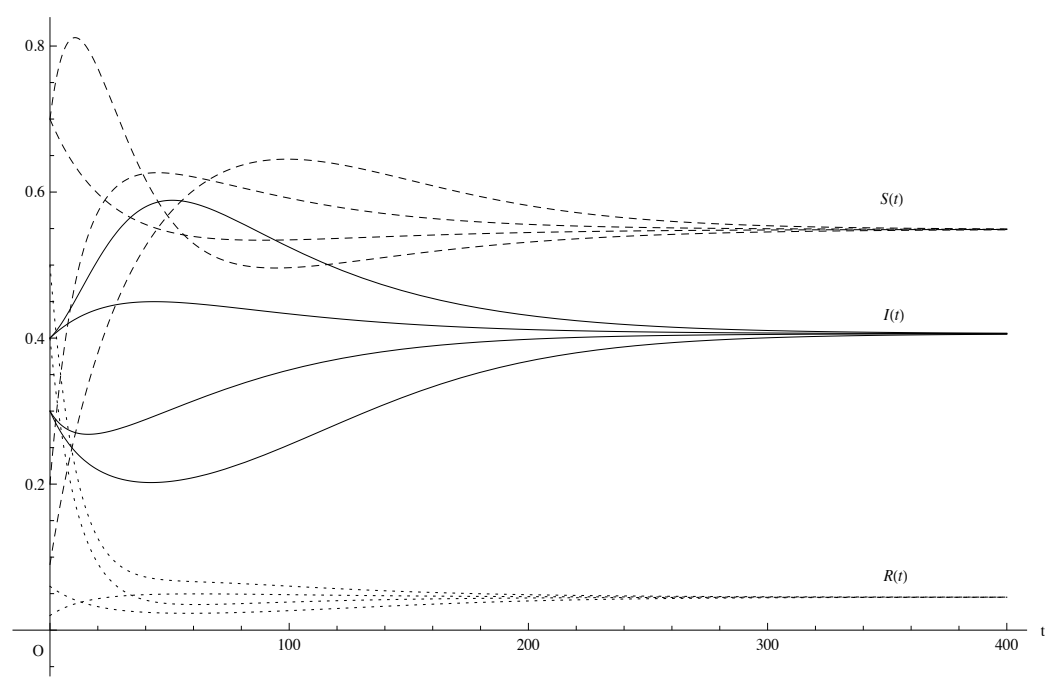

Fig. 3 The graph trajectory of $S(t), I(t)$ and $R(t)$ of system (5.1). For the case (5.3) with $\beta=0.06$ and $\delta=0.07 \geq \delta^{*}$, we have $R_{0}=1.818 \cdots>1$ and $E^{*}=(0.548 \cdots, 0.406 \cdots, 0.045 \cdots)$.

\section{Discussion}

In this paper, for an SIRS epidemic model with a wide class of nonlinear incidence rates and distributed delays $\int_{0}^{h} p(\tau) f(S(t), I(t-\tau)) d \tau$, we established the global asymptotic stability of the disease-free equilibrium $E_{0}$ for $R_{0} \leq 1$ and the endemic equilibrium $E_{*}$ of system (1.2) for $R_{0}>1$. By using a limit system of the model, a proof that the disease-free equilibrium is globally asymptotically stable for $R_{0}=1$ is also given. In particular, without imposing any restriction on the size of a maximum latent period $h$, the global asymptotic stability of the endemic equilibrium $E_{*}$ of system (1.2) is established for a small loss of immunity rate $\delta$. By means of strict monotonicity of functions $f(S, I)$ and $f(S, I) / I$ with respect to $S \geq 0$ and $I>0$, we obtain Lemma 4.1 which plays an important role to establish the condition (1.5) such that $V$ is a Lyapunov functional. Hence, our result is an extension to the global stability result for an SIR epidemic model.

\section{Acknowledgements}

The authors wish to express their gratitude to the editor and an anonymous referee for very helpful comments and valuable suggestion which improved the quality of this paper. The authors' work was supported in part by JSPS Fellows, No.237213 of Japan Society for the Promotion of Science to the first author, by the Grant MTM2010-18318 of the MICINN, Spanish Ministry of Science and Innovation to the third author, and by Scientific Research (c), No.21540230 of Japan Society for the Promotion of Science to the fourth author.

\section{References}

1. M.E. Alexander and S.M. Moghadas, Bifurcation analysis of an SIRS Epidemic model with generalized incidence, SIAM J. Appl. Math. 65 (2005) 1794-1816. 
2. E. Beretta, T. Hara, W. Ma and Y. Takeuchi, Global asymptotic stability of an SIR epidemic model with distributed time delay, Nonlinear Anal. 47 (2001) 4107-4115.

3. V. Capasso and G. Serio, A generalization of the Kermack-Mckendrick deterministic epidemic model, Math. Biosci. 42 (1978) 43-61.

4. K.L. Cooke, Stability analysis for a vector disease model, Rocky Mountain J. Math. 9 (1979) 31-42.

5. Y. Enatsu, Y. Nakata and Y. Muroya, Global stability of SIR epidemic models with a wide class of nonlinear incidence rates and distributed delays, Discrete Continuous Dynamical System, Series B 15 (2011) 61-74.

6. Y. Enatsu, Y. Nakata and Y. Muroya, Global stability of SIRS epidemic models with a class of nonlinear incidence rates and distributed delays, To appear.

7. G. Huang, Y. Takeuchi, W. Ma and D. Wei, Global stability for delay SIR and SEIR epidemic models with nonlinear incidence rate, Bull. Math. Biol. 72 (2010) 1192-1207.

8. G. Huang and Y. Takeuchi, Global analysis on delay epidemiological dynamic models with nonlinear incidence, J. Math. Biol. doi:10.1007/s00285-010-0368-2.

9. Y. Jin, W. Wang and S. Xiao, An SIRS model with a nonlinear incidence rate, Chaos, Solitons and Fractals 34 (2007) 1482-1497.

10. A. Korobeinikov, P.K. Maini, Nonlinear incidence and stability of infectious disease models, Math. Med. Biol. 22 (2005) 113-128.

11. A. Korobeinikov, Lyapunov functions and global stability for SIR and SIRS epidemiological models with non-linear transmission, Bull. Math. Biol. 68 (2006) 615-626.

12. A. Korobeinikov, Global Properties of Infectious Disease Models with Nonlinear Incidence, Bull. Math. Biol. 69 (2007) 1871-1886.

13. Y. Kuang, Delay Differential Equations with Applications in Population Dynamics, Academic Press, San Diego, 1993.

14. C.C. McCluskey, Complete global stability for an SIR epidemic model with delay-Distributed or discrete, Nonl. Anal. RWA. 11 (2010) 55-59.

15. C.C. McCluskey, Global stability for an SIR epidemic model with delay and nonlinear incidence, Nonl. Anal. RWA. 11 (2010) 3106-3109.

16. C.C. McCluskey, Global stability of an SIR epidemic model with delay and general nonlinear incidence, Math. Biosci. Engi. 7 (2010) 837-850

17. J. Mena-Lorcat and H.W. Hethcote, Dynamic models of infectious diseases as regulators of population size, J. Math. Biol. 30 (1992) 693-716.

18. Y. Muroya, Y. Enatsu and Y. Nakata, Global stability of a delayed SIRS epidemic model with a nonmonotonic incidence rate, J. Math. Anal. Appl. 377 (2011) 1-14.

19. Y. Muroya, Y. Enatsu and Y. Nakata, Monotone iterative techniques to SIRS epidemic models with nonlinear incidence rates and distributed delays, Nonl. Anal. RWA. 12 (2011) 1897-1910.

20. Y. Nakata, Y. Enatsu and Y. Muroya, On the global stability of an SIRS epidemic model with distributed delays, To appear.

21. Y. Takeuchi, W. Ma and E. Beretta, Global asymptotic properties of a delay SIR epidemic model with finite incubation times, Nonlinear Anal. 42 (2000) 931-947.

22. C. Vargas-De-León and G. Gómez-Alcaraz, Global stability conditions of delayed SIRS epidemiological model for vector diseases, Foro-Red-Mat: Revista Electrónica de Contenido Matemático 28 (2011)

23. R. Xu and Z. Ma, Stability of a delayed SIRS epidemic model with a nonlinear incidence rate, Chaos, Solitons and Fractals 41 (2009) 2319-2325.

24. X. Zhou and J. Cui, Analysis of stability and bifurcation for an SEIV epidemic model with vaccination and nonlinear incidence rate, Nonlinear Dynamics 63 (2011) 639-653. 